\title{
OPTICALLY DRIVEN RESONANT MICROBEAM TEMPERATURE SENSORS FOR FIBER OPTIC NETWORKS
}

\author{
D. W. Burns, W. R. Herb, J. D. Zook and M. L. Wilson \\ Honeywell Technology Center \\ 12001 State Highway 55 \\ Plymouth, Minnesota 55441-4799
}

\begin{abstract}
Fiber optic sensors combining micromachined polysilicon resonators with all-optical drive and sense techniques have been demonstrated for single-point and multi-point measurements of temperature using an optical fiber network. Sealed cavity resonators, varying from $122 \mu \mathrm{m}$ to $324 \mu \mathrm{m}$ in length, are integrally fabricated on a thin, single crystal silicon paddle with a thermal expansion mismatched film on the backside. Bending of the bimorph due to differential expansion with temperature alters the resonant frequency of the microbeam. The microbeam is driven photovoltaically with an embedded photodiode under the microbeam, and the resonance is detected by modulation of the reflected light as the microbeam vibrates. A vibration amplitude of $20 \mathrm{~nm}$ can modulate the reflected light by as much as $50 \%$.

Each resonant microbeam is designed to operate within a prescribed frequency band, providing four to sixteen nodes of temperature sensing in a linear network with one microbeam at each node. Scanning, pulse-and-ringdown and self-resonant methods have been used to detect the microbeam frequencies. Individual devices have been demonstrated with a sensitivity up to $265 \mathrm{~Hz} /{ }^{\circ} \mathrm{C}$ depending on paddle and bimorph thickness. Noise measurements on self-resonant microbeams have shown root Allan variances as low as $0.014 \mathrm{~Hz}$, corresponding to a temperature resolution of $10^{-4} \mathrm{~K}$. Hysteresis and repeatability measurements are currently underway. The devices have been packaged and operated successfully between $0^{\circ} \mathrm{C}$ and $200^{\circ} \mathrm{C}$. Pressure sensors, vibration sensors, accelerometers, strain gages, acoustic emission sensors and other mechanical sensors can be configured using an appropriate silicon microstructure and attached to the optical fiber network.
\end{abstract}

\section{INTRODUCTION}

Honeywell is developing fiber optic sensors utilizing silicon micromachined, sealed cavity resonant microbeam technology initially developed at the University of Wisconsin [1] to meet the need for single-point and multi-point distributed optical sensor networks. An optical resonant microbeam sensor consists of a micromachined silicon chip attached to a multimode optical fiber. Light from an external source excites the beam into resonance, and vibrations of the microbeam modulate the light reflected back into the fiber which is detected by an external photodetector. We have previously demonstrated optical drive and sense using light from a semiconductor laser $[2,3]$, and more recently reported the demonstration of optically excited self-resonance with unmodulated laser light [4]. Strain and pressure sensitivity have also been demonstrated, both at the wafer level and in pressure sensitive fixtures. Honeywell is currently teamed with the University of Wisconsin and Mobil Oil to develop a class of devices for fiber optic sensors and networks. These STORM (Strain Transduction by Optically Resonant Microbeams) devices are specifically aimed at precision temperature measurement for use in remote, hazardous or high EMI environments where advantages exist over inexpensive thermocouples and RTDs.

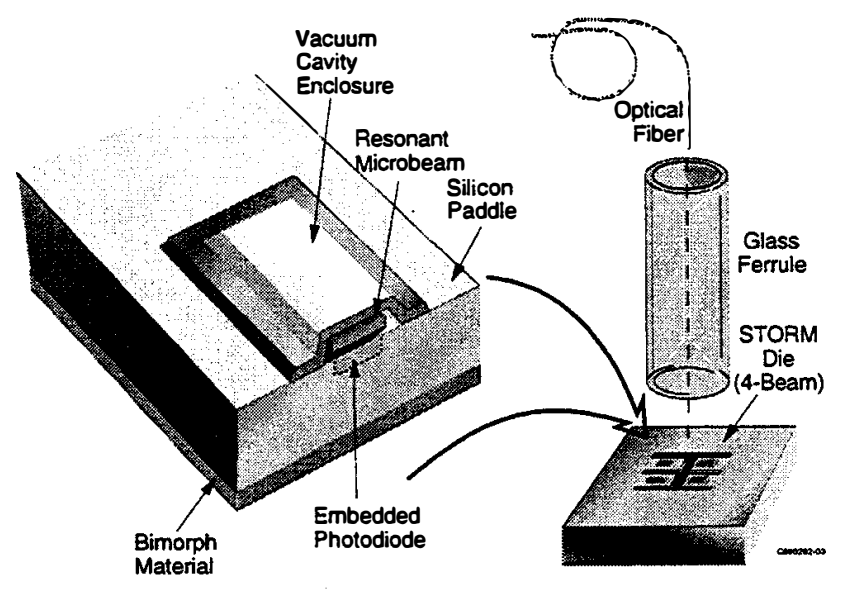

Figure 1. STORM temperature sensor die with ferrule and fiber. The STORM chip contains sealed cavity resonant microbeam strain sensors on a suspended bimorph of silicon and a thermally mismatched material. The optical fiber and glass ferrule are selectively aligned to a microbeam on the STORM temperature sensor die. Light transmitted over the fiber is used to excite the microbeam and detest its resonant frequency, which shifts appreciably with temperature.

The STORM temperature sensor consists of an optically excited, optically detected resonant microbeam on a silicon die which has been micromachined to form single crystal silicon "paddles" on which a thermal coefficient of expansion (TCE) mismatched material is deposited (see Figure 1).

The resonators are fabricated from thin films of fine-grain LPCVD polysilicon between 0.8 and $2.4 \mu \mathrm{m}$ thick, and are enclosed in a vacuum cavity enclosure formed by a polysilicon shell. The gaps between the microbeam and enclosure, on the order of $0.5 \mu \mathrm{m}$, are accurately dimensioned based on design and modeling. The vacuum cavity allows the microbeam to vibrate freely at its resonant frequency, unaffected by the medium outside the integral enclosure. The silicon paddles isolate the microbeams from package-induced strains. A large shift in the resonant frequency of the microbeam occurs when strained by temperature-induced bending of the bimorph. An optical fiber with a $50 \mu \mathrm{m}$ core is held in a simple glass ferrule which is fastened to the die. Incident light at a specified wavelength (ie. $780 \mathrm{~nm}$ ) is partially transmitted through the shell and microbeam, and absorbed by the underlying photodiode. A photovoltage is generated which attracts the microbeam downwards. Vibrations of the resonant microbeam cause intensity modulation of reflected and transmitted light. The incident light is partially reflected from each surface (shell, microbeam and substrate) and collected by the same optical fiber. The microbeam vibration changes the modulation index for the reflected light. A $3 \mathrm{db}$ coupler is used in conjunction with a simple photodetector to measure the reflected light and determine the resonant frequency of the microbeam. Also, with proper choice of cavity and microbeam thickness, unmodulated light can be used to excite the microbeam to self-resonance [4]. 


\section{MECHANICAL DESIGN}

The design of the STORM temperature sensor involves electrical, optical, mechanical and structural considerations. The electrical considerations are minimal, requiring only that one side of the photodiode be ohmically connected to the resonant microbeam and that the photodiode has an appropriate carrier lifetime. Ohmic connection is obtained by implanting the microbeam with the same type ( $n$ or $p$ ) as the substrate it resides on, with the photodiode formed by implanting the opposite species prior to microbeam formation. The optical considerations are critical and are discussed in the next section. Mechanical considerations are made to maximize the signal within constraints imposed by the processing to avoid parasitic resonances and mechanical coupling, and allow for packaging with minimal package stress effects.

The temperature-sensitive bimorph is cantilevered which allows unconstrained deflections and prevents undesirable package stresses and hysteresis from affecting the performance. The strain $\varepsilon_{\text {app }}$ induced at the surface of the paddle is uniform along its length [5] and is given approximately by (for $\left.\mathrm{h}_{2}<<\mathrm{h}_{1}\right)$

$$
\varepsilon_{\text {app }}=3 \Delta T \Delta \alpha \frac{\left(h_{1}+h_{2}\right) h_{2}}{h_{1}^{2}}\left(\frac{E_{2}}{1-v_{2}}\right)\left(\frac{1-v_{1}}{E_{1}}\right)
$$

where $\Delta \mathrm{T}$ is the temperature shift, $\Delta \alpha$ is the TCE mismatch, $\mathrm{h}_{1}$ and $h_{2}$ are the thickness of the silicon paddle and bimorph material, respectively, and $E$ and $v$ are the elastic moduli and Poisson's ratio. The dependence of the resonant frequency of a microbeam on applied strain is given by

$$
f_{r}=\frac{b_{n}}{2 \pi}\left(\frac{E}{\rho}\right)^{1 / 2}\left(\frac{h_{b}}{l_{b}{ }^{2}}\right)\left[1+\gamma_{n}\left(\frac{l_{b}}{h_{b}}\right)^{2}\left(\varepsilon_{o}+\varepsilon_{a p p}\right)\right]^{1 / 2}
$$

where $E$ and $\rho$ are Young's Modulus and density, $h_{\mathrm{b}}$ and $l_{\mathrm{b}}$ are the beam thickness and length, $E$ is the intrinsic strain, and $b_{\mathrm{n}}$ and $\gamma_{n}$ are coefficients with values 6.4586 and 0.2949 for the fundamental mode [6]. The temperature sensitivity of the microbeam may then be found by combining equations (1) and (2). If intrinsic strain in the microbeam is neglected, the temperature sensitivity may be expressed as:

$$
\frac{\Delta f}{\Delta T}=\left(\frac{3 \gamma_{n}}{2}\right) \Delta \alpha \frac{\left(h_{1}+h_{2}\right) h_{2}}{h_{1}^{2}}\left(\frac{l_{b}}{h_{b}}\right)^{2}\left(\frac{E_{2}}{1-v_{2}}\right)\left(\frac{1-v_{1}}{E_{1}}\right)
$$

From this equation it can be seen that the sensitivity increases with TCE mismatch and thickness of the bimorph as expected. The microbeam itself is mildly temperature sensitive: due to small density changes and a slight softening of the elastic moduli, a temperature sensitivity of about $-40 \mathrm{ppm} /{ }^{\circ} \mathrm{C}$ can be added to the result of equation (2). The TCE-mismatched material can be placed on either side of the silicon paddle, though for expansion coefficients larger than that of silicon, the bimorph material should be placed on the backside. An additional film with a smaller TCE than silicon such as silicon nitride can be placed on the front, which passivates the microbeam and forms an antireflection coating. Consequently, the output frequency will have a negative coefficient.

ANSYS modeling was used to verify and further guide the design. Of particular concern is the avoidance of structural resonances of the paddles or die. Flexural or torsional modes of the cantilevered paddle can couple with microbeam motion, causing a loss in vibration amplitude and gaps in the frequency response. A plot of the structural modes for four microbeams on individual paddles is shown in Figure 2, with nonoverlapping microbeam modes avoiding the paddle modes.

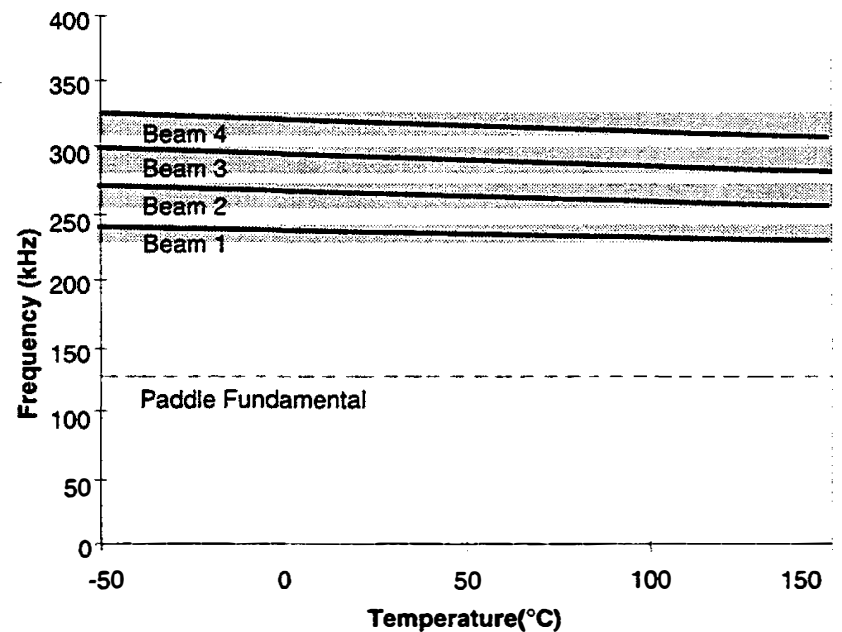

Figure 2. Modal analysis plot of four-beam STORM die. The structural modes of the paddles have been designed to be well above and below the microbeam frequencies to avoid unwanted mechanical coupling.

Mechanical modeling was also done on an alternative support design for the resonant microbeam. This design allows reduction of the thermal cycles during microbeam processing by using a deposited sacrificial layer rather than a thermally grown oxide. This microbeam design is supported by small posts formed by opening windows in the lower sacrificial layer prior to deposition of the beam poly, avoiding non-planar features spanning the entire beam width. The posts support the microbeam and provide ample coupling of applied strain to the ends (see Figure 5). ANSYS modeling shows minimal frequency variations with narrow or wide posts, or varying number of posts. Calculations also show little shift in the gage factor with varying post width. Most importantly, the new microbeam supports result in minimal static deflection of the free-standing microbeam with non-zero internal or applied strain, resulting in planar microbeams and consistent gaps with respect to the substrate and shell.

\section{OPTICAL DESIGN}

The design of optically resonant microbeam strain transducers has been described previously for optically driven microbeams [3] and self-resonant microbeams [4]. Although self-resonance has the attractive feature of not requiring laser modulation, it can easily produce optical fringe folding resulting in strong harmonic generation. Therefore for the optical network sensors, an optical design was chosen that has negative internal feedback rather than the positive internal feedback that leads to self-resonance. In either case, the microbeam thickness is chosen to be an odd number of quarter waves so that the rays reflected from the upper and lower surface interfere constructively to produce maximum modulation of the reflected light. The sum of the thicknesses of the two cavities above and below the microbeam is a multiple of a half wave for the same reason. Self-resonance can be avoided by simply interchanging the thicknesses of the upper and lower cavity so that the total reflectivity increases, rather than decreases, as the beam moves away from the substrate. With the proper choice of cavity and microbeam thicknesses, the reflectivity can be high and still readily modulated by the microbeam vibration over a fairly wide wavelength region. Calculated reflectivity as a function of microbeam position is illustrated in Figure 3, which shows a $20 \%$ modulation of the reflected light for a microbeam amplitude of $10 \mathrm{~nm}$. 


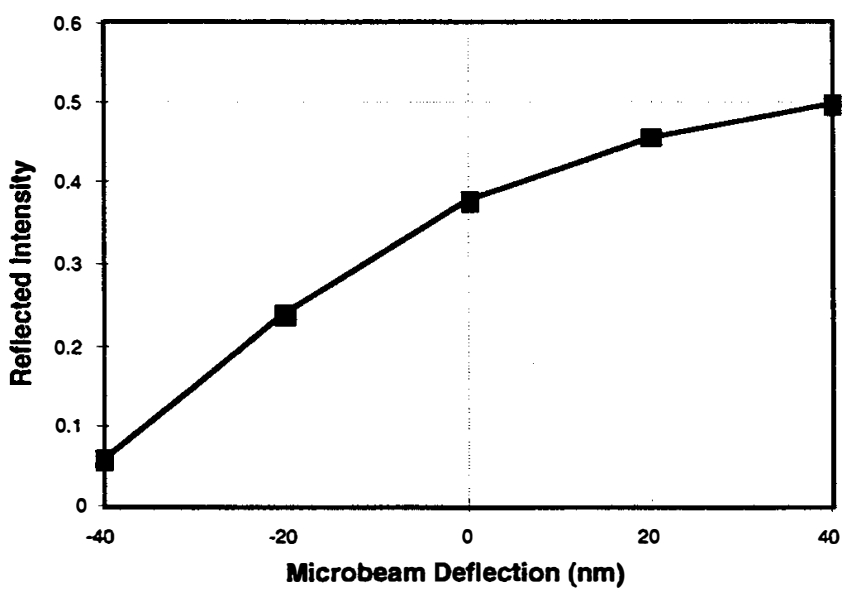

Figure 3. Reflectivity as a function of microbeam position for a case where the cavity thicknesses are 445 and $335 \mathrm{~nm}$, and the incident wavelength is $780 \mathrm{~nm}$. The microbeam and shell thicknesses are $2.26 \mu \mathrm{m}$ and $2.16 \mu \mathrm{m}$, respectively.

\section{DIE DESIGN AND FABRICATION}

Four STORM die were designed with multiple microbeam sensors per die, each having a different resonant frequency for use on a different node of the network. Two temperature sensor die have four microbeams on four individual paddles, arranged in a 2x2 array; one with enlarged circular regions and circular photodiodes, the other with rectangular microbeams and elongated photodiodes. A sixteen-beam temperature and a threebeam pressure sensor complete the set. A plot of the four-beam device is shown in Figure 4.

The microbeam fabrication sequence uses six photomasks and begins with a deposition of Low Temperature Oxide (LTO) which is patterned and etched to form the post regions at the ends of the microbeams. An implant is done through the LTO using a photoresist mask to form a shallow photodiode directly under each microbeam. The microbeam is then formed by depositing LPCVD polysilicon, implanting it to provide ohmic contact to the substrate and removing the poly in the field region away from the microbeams. A second LTO layer is deposited and etched in ring-shaped regions around each microbeam to expose the substrate prior to deposition of the shell poly. A thin LTO layer is deposited and patterned in the ring region to form etch channels for subsequent sacrificial etching. The shell poly is deposited, implanted and patterned to cover each microbeam. A hydrofluoric acid solution is used as a sacrificial etchant to etch away the LTO in the field, in the etch channel regions, and in the regions immediately above and below the microbeam. Following carefully selected rinse cycles, a modification of the sublimation sequence previously reported [7] is used to freeze the final rinse fluid, which is then pumped out at reduced temperatures to produce free-standing beams. A final LPCVD film is deposited to seal the vacuum cavities as the thin etch channels grow shut, followed by a thin layer of silicon nitride that further enhances the seal and provides an antireflection coating. No metal depositions are used in the process sequence.

Microstructure formation follows microbeam fabrication and uses an $\mathrm{SF}_{6}$-based RIE etch to form trenches around the microbeams. The microbeams and trenches are passivated with an additional layer of LTO to provide protection during the subsequent anisotropic etch. A KOH or EDP etchant is used after patterning the oxide on the backside of the wafer to locally thin the wafers, followed by removal of the passivation LTO to free the silicon paddles. A thin film of TCE-mismatched material is

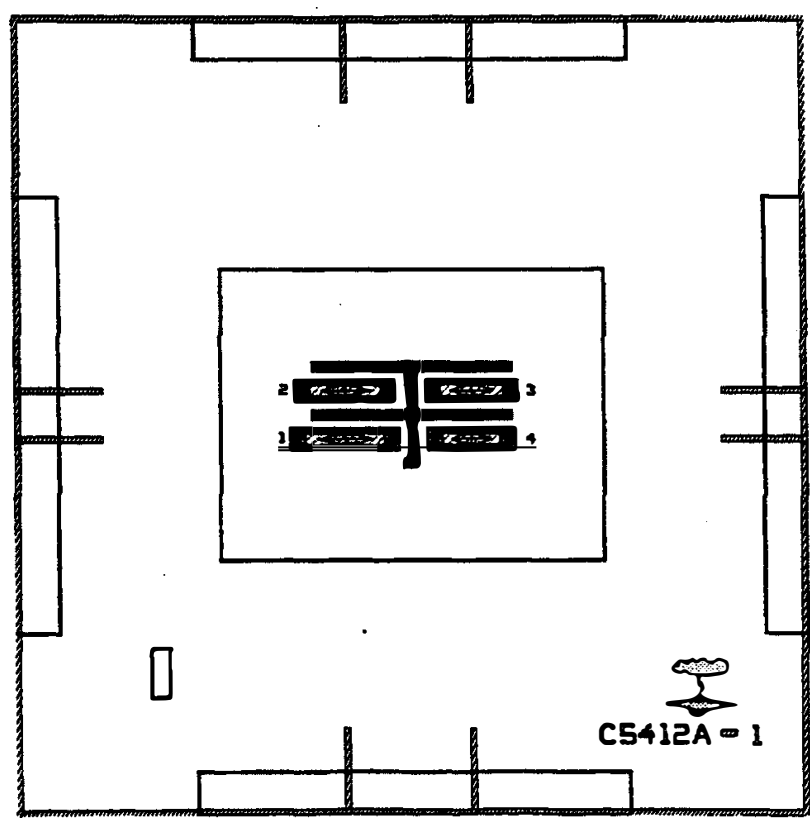

Figure 4. Four-beam STORM temperature sensor die. An array of microbeams ranging from $240 \mu \mathrm{m}$ to $324 \mu \mathrm{m}$ long are formed on individual paddles $400 \mu \mathrm{m}$ long. Deep trenches are formed around the paddles, and a backside anisotropic etch is used to locally thin the silicon. The die footprint is $3.3 \mathrm{~mm} x$ $3.3 \mathrm{~mm}$.

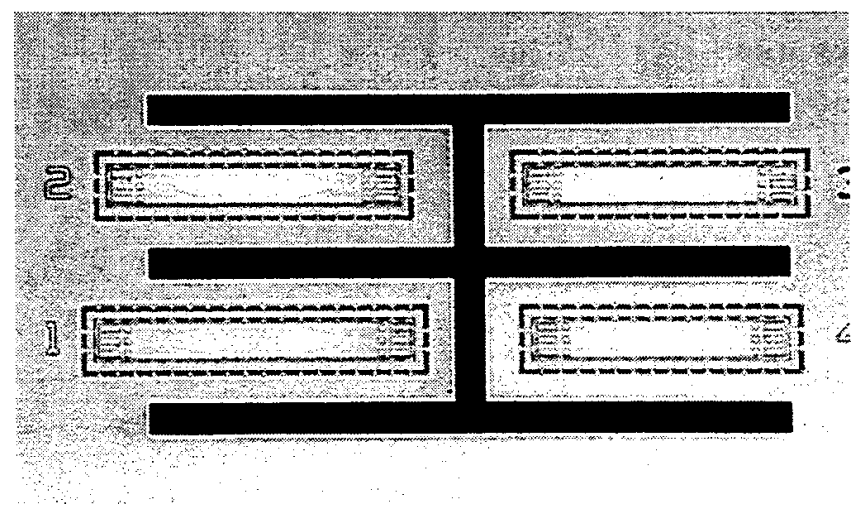

Figure 5. Optical micrograph of STORM temperature sensor die. Four microbeams are located on individual silicon paddles and operate at different frequencies. Close inspection reveals the post-style supports and the elongated embedded photodiodes.

deposited from the backside of the wafer onto the paddles to form the bimorph. A completed four-microbeam die is shown in Figure 5.

\section{PACKAGING AND TESTING}

The package currently being utilized for the STORM temperature sensors consists of a STORM die, glass ferrule with funnel-shaped ends to hold an optical fiber, and a metal housing (see Figure 1). The glass tube containing the optical fiber is attached to the STORM die. The tube has a thermal expansion coefficient similar to silicon and provides excellent stabilization of the end of the fiber with respect to the STORM chip. A fixture has been developed to aid in the packaging, 


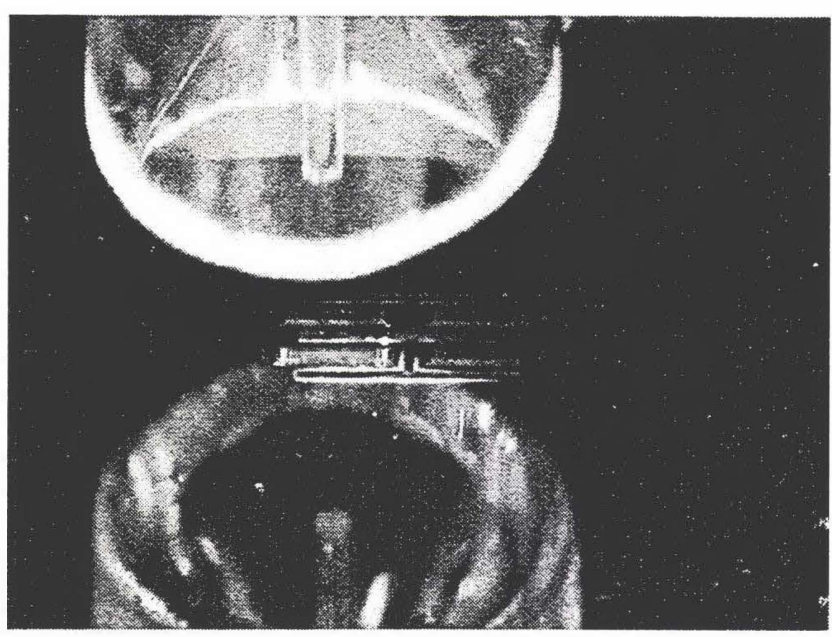

Figure 6. STORM packaging fixture. An optical fiber and glass ferrule are seen directly and reflected in a STORM 4-beam die as viewed through a microscope. Magnetic clamps hold the fiber and ferrule while vacuum holds the STORM die. Coarse alignment is achieved visually followed by fine adjustment using active alignment.

consisting of a video camera and monitor to allow the operator to visually align the optical fiber to the appropriate microbeam. Active alignment by monitoring the microbeam resonance is used for fine position adjustment of the optical fiber with independent $x, y$ and $z$-axis control. The STORM chip is held with a vacuum chuck that can be electrically heated to $250^{\circ} \mathrm{C}$ so that solders or thermal set adhesives can be used to bond the glass ferrule and silicon die. The assembly is placed in a threaded metal package for external attachment and a strain relief for the pigtailed fiber. Current packages are $5 \mathrm{~mm} \times 5 \mathrm{~mm}$ x $15 \mathrm{~mm}$, but smaller packages are planned.

Testing of the STORM devices has been done at the wafer level and in assembled packages. During wafer level testing, the incident light strikes the microbeam at an angle and a second fiber is used to collect the reflected light. Resonant peaks are detected using a spectrum analyzer or a gain-phase analyzer. A plot of resonant frequencies from a sixteen-beam STORM die is shown in Figure 7. Resonant frequencies on test chips varied from $197 \mathrm{kHz}$ for a $400 \mu \mathrm{m}$ long beam to 1.17 $\mathrm{MHz}$ for a $100 \mu \mathrm{m}$ long beam. Q values as high as 100,000 were measured on some beams. A hot chuck has been used to measure the temperature shift of the resonant microbeams. The first device tested had a base frequency of $207.5 \mathrm{kHz}$ with a frequency sensitivity of $-115 \mathrm{~Hz} /{ }^{\circ} \mathrm{C}$. A four-beam sensor has also been demonstrated, with baseline frequencies of 207.7 $\mathrm{kHz}, 250.4 \mathrm{kHz}, 297.4 \mathrm{kHz}$ and $340.6 \mathrm{kHz}$. The corresponding temperature sensitivities are between -112 $\mathrm{Hz} /{ }^{\circ} \mathrm{C}$ and $-118 \mathrm{~Hz} /{ }^{\circ} \mathrm{C}$ for each beam. Higher sensitivities were obtained by depositing additional material on the back of the paddle, with sensitivities increasing linearly with material thickness, approaching $-265 \mathrm{~Hz} /{ }^{\circ} \mathrm{C}$. A sensitivity of -30 $\mathrm{Hz} /{ }^{\circ} \mathrm{C}$ was measured with only a silicon nitride passivation layer. Unpackaged die have been used to establish operating temperature ranges above $200^{\circ} \mathrm{C}$ (see Figure 8). Although noise measurements are yet to be made on networked sensors, measurements have been taken on optically-coupled selfresonant microbeams. An RAV of $0.014 \mathrm{~Hz}$ was measured at a sample time of 0.3 seconds, indicating that temperature resolution less than $100 \mu \mathrm{K}$ is attainable with the optically interfaced sensors. A laser vibrometer has also been used to measure the structural resonances of the paddles [6], which are also in good agreement with the ANSYS modeling.

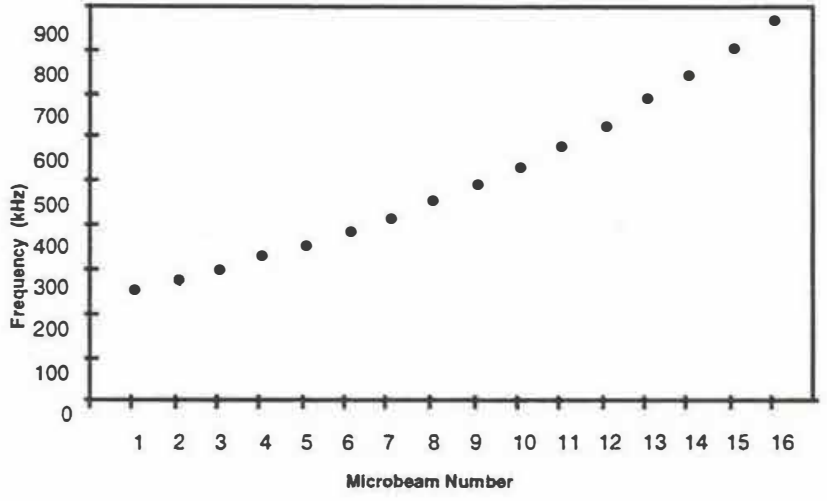

Figure 7. Measured resonances on a sixteen-beam sensor die. The sixteen frequencies vary from $251 \mathrm{kHz}$ to $865 \mathrm{kHz}$ (room temperature) for the $1.102 \mu \mathrm{m}$ thick microbeams with signal and guard bands of approximately $10 \%$ between microbeams.

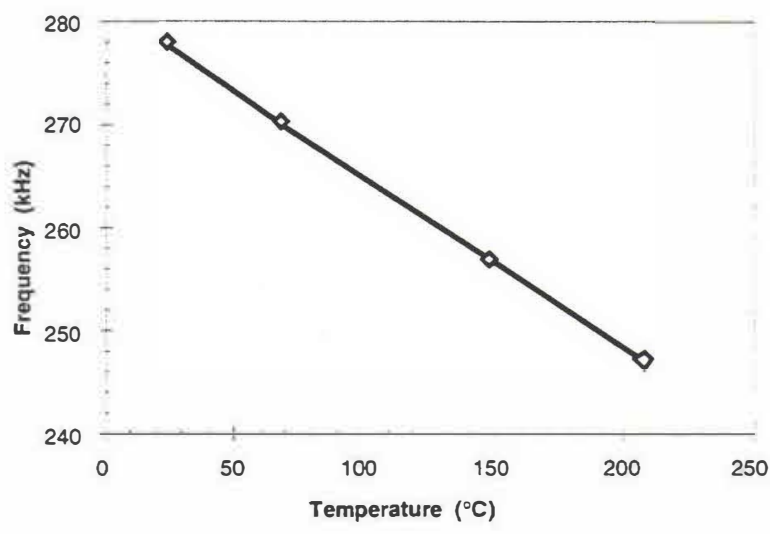

Figure 8. Frequency output of a single resonant microbeam with temperature. A $30 \mathrm{kHz}$ shift was obtained over a $175^{\circ} \mathrm{C}$ temperature range for a STORM die mounted on a hot chuck. Operation above $200^{\circ} \mathrm{C}$ has been demonstrated.

\section{OPTICAL NETWORKS}

Two different network topologies have been investigated, one based upon a lightly tapped linear bus, and the second based upon a star bus. An advantage of a linear tapped bus is that a single fiber can be used to connect the entire network. However, this can also be a disadvantage if the fiber is damaged or broken since all sensors downstream from the break would no longer function. The star bus has the advantage that the signal levels are higher and there is no single point failure mechanism except if the star coupler is damaged.

Figure 9 illustrates the star bus design. It is possible to use several different wavelength lasers together with wavelength filters at the STORM chip in order to use the same resonant frequency several times on the bus. Thus the combination of Wavelength Division Multiplexing (WDM) with Frequency Division Multiplexing (FDM) techniques allows for the connection of much larger optical sensor networks.

The signal obtained from an actual STORM device is shown in Figure 10. The noise floor is at $-90 \mathrm{dBm}$, and the signal is about $+45 \mathrm{~dB}$ above the noise floor. The signal to noise ratio (SNR) of this signal is very large making signal processing much easier. In Figure 10, the spectrum analyzer's filter bandwidth is convolved with this resonant peak, so the actual peak is much sharper than shown in this figure. Figure 11 shows data obtained from a small $2 \times 2$ star network similar to 


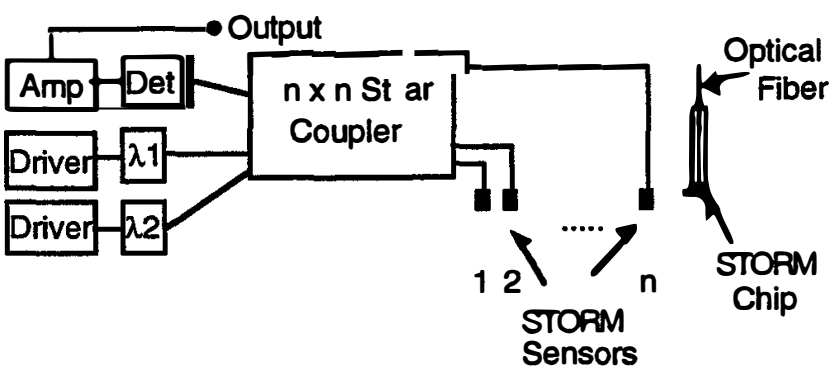

Figure 9. Optical fiber network block diagram. One to $n$ STORM die are attached to system optics using an $n \times n$ star coupler.

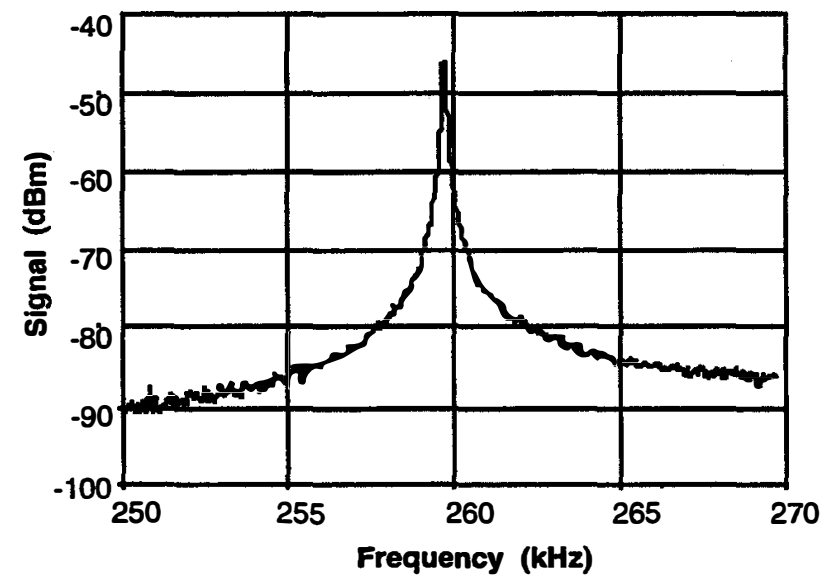

Figure 10. The resonant frequency spectrum of a STORM device.

that detailed in Figure 9. Two temperature sensors each with different resonant frequencies were connected to this network. Note that each frequency is free from inter-modulation harmonics and each peak has an excellent SNR.

\section{CONCLUSIONS AND FUTURE DIRECTIONS}

STORM sensors represent a new and novel optoelectromechanical sensor technology which combines the strength of silicon resonant microbeam technology - including high sensitivity, stability and robustness - with that of optical fiber technology - including EMI immunity and intrinsic safety. The STORM technology provides the generic basis for a wide range of sensors that offer significant advantages over conventional silicon-only or fiber-only sensors. Fiber optic networks for silicon-based resonant sensors are being demonstrated, with first applications to temperature sensing. The resonant microbeams are highly sensitive to axial strain and can be configured with suitable silicon microstructures to form precision pressure sensors, accelerometers, force sensors, vibration sensors, rotation sensors and load cells as well as temperature sensors, with possibilities of multiple sensors per node if desired. Optical fiber interfacing to resonant microbeam sensors can be achieved without deterioration of the output signals due to EMI, no loss in ambiguity as in many interference-based sensors and without any on-chip electronics. The limits of networkability have not been firmly established, though a variety of bus architectures appear feasible. Further development of the optically self-resonant and non-selfresonant concepts are needed to address questions relevant to producibility, fiber-to-chip coupling and tolerances on optical components.

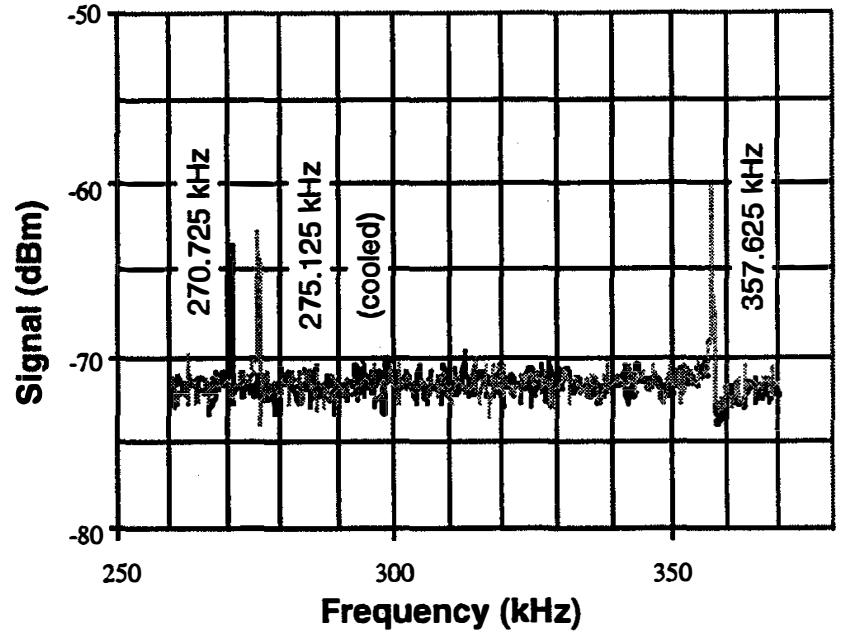

Figure 11. Output from a sensor network with two devices. Two overlapping scans are shown, one taken later in time with (only) one node reduced in temperature and showing a frequency shift of $4400 \mathrm{~Hz}\left(\Delta T=-30^{\circ} \mathrm{C}\right)$. The sensors were separated by several meters of optical fiber, joined by a four-port coupler.

\section{ACKNOWLEDGMENTS}

The authors would like to gratefully acknowledge Dane Larson and Jerry Huber for sensor processing, Cindy Bassett, Emest Satren and Jerry Bolser for sensor testing and packaging, Hirak Chanda for FEM calculations, Peg Wilson for layout, Barry Cole for optical modeling, Professor Guckel at the University of Wisconsin for his support and technical contributions, and ARPA (contract \#DAAL01-94-C-3427) for partial funding of this effort.

\section{REFERENCES}

1. J. D. Zook, D. W. Burns, H. Guckel, J. J. Sniegowski, R. L. Engelstad and Z. Feng, "Characterization of Polysilicon Resonant Microbeams," Sensors and Actuators A, Vol. 35 (1992), pp. 51-59.

2. H. Guckel, M. Nesnidal, J. D. Zook and D. W. Burns, "Optical Drive/Sense for High Q Resonant Microbeams," Technical Digest of the 7th International Conference on SolidState Sensors and Actuators, pp. 686-689.

3. J. D. Zook, D. W. Bums, J. N. Schoess and H. Guckel, "Optically Resonant Microbeams," SPIE, Photonics West '95, Feb. 10, 1995.

4. J. D. Zook, D. W. Burns, W. R. Herb, H. Guckel, J.-W. Kang and Y. Ahn, "Optically Excited Self-Resonant Strain Transducers," Transducers '95, pp. 600-603.

5. S. Timoshenko, "Analysis of Bi-Metal Thermostats," J. of the Optical Society of America, Vol. 11, 1925, pp. 233-255.

6. D. W. Burns, J. D. Zook, R. D. Homing, W. R. Herb and H. Guckel, "Sealed-Cavity Resonant Microbeam Pressure Sensors," Sensors and Actuators A, Vol. 48 (1995), pp. 179-186.

7. H. Guckel, J. J Sniegowski, T. R. Christenson and F. Rassi, "Te Application of Fine-Grained, Tensile Polysilicon to Mechanically Resonant Transducers," Sensors and Actuators, A21-A23 (1990), pp. 346-351. 